\title{
The challenge of mass conservation in the solution of free-surface flows with the fractional-step method: Problems and solutions
}

\author{
Sergio R. Idelsohn $*, \dagger, \ddagger$ and Eugenio Oñate ${ }^{\S}$ \\ International Centre for Numerical Methods in Engineering (CIMNE), Universidad Politénica de Cataluña (UPC), \\ Campus Nord UPC, 08034 Barcelona, Spain
}

\begin{abstract}
SUMMARY
The purpose of this paper is to put in evidence that the fractional-step method (FSM) used to solve the incompressible transient Euler and Navier-Stokes equations for free-surface flows has a problem inherent to the method that may produce unacceptable variations of the domain volume. A simple modification of the free-surface boundary term is introduced in order to reduce considerably the volume loss and preserve the computational advantages of the FSM. Copyright (c) 2008 John Wiley \& Sons, Ltd.
\end{abstract}

Received 22 July 2008; Revised 18 November 2008; Accepted 24 November 2008

KEY WORDS: free-surfaces; fractional-step method; Navier-Stokes equations; mass conservation

\section{INTRODUCTION}

The fractional-step method (FSM), proposed first by Chorin $[1,2]$ in the $1960 \mathrm{~s}$, is probably one of the most popular techniques to solve the incompressible transient equations of fluid mechanics.

The advantages of the FSM are well known:

1. Separation of the pressure from the velocity unknowns.

2. For some particular values of the time step increment, the FSM gives stable results for equal-order elements without any stabilization term.

3. For the Euler equations, or even for a small viscosity value, the momentum equations may be solved for each velocity component in an uncoupled manner.

Each of these advantages justifies the use of the FSM for many fluid mechanic problems:

1. Separation of the pressure unknown from the velocity not only reduces the total number of degrees of freedom (DOFs) to be solved simultaneously, but it is advantageous for the characteristic and quality of the non-linear coupled system of equations. This system has a very low condition number, which is put it in evidence when using any iterative scheme $[3,4]$.

\footnotetext{
${ }^{*}$ Correspondence to: Sergio R. Idelsohn, International Centre for Numerical Methods in Engineering (CIMNE), Universidad Politénica de Cataluña (UPC), Campus Nord UPC, 08034 Barcelona, Spain.

†E-mail: sergio@cimne.upc.edu, http://www.cimne.com

${ }^{\ddagger}$ ICREA Research Professor.

${ }^{\S}$ Professor.
}

Contract/grant sponsor: SEDUREC project of the Consolider Injenio 2010 programme of the Minister of Science and Education of Spain 
2. CBS methods [5] are based on the stabilization introduced by the FSM on the pressure unknown. Nevertheless this advantage is not so clear because the stabilization disappears or becomes insufficient when the time step is small $[3,4]$.

Finally, the possibility to solve the momentum equations separately for each velocity component is a big advantage because it is computationally more efficient to solve $3 n \times n$ systems of equations that a system of $3 n \times 3 n$ equations, being $n$ the number of nodes. Note however that the possibility of splitting the equations for viscous free-surface flows is not so evident. For viscous flows there are some boundary terms that are coupled for the three velocity components [6]. However, in this case it is also possible to treat explicitly the terms that do not contribute to the diagonal of the viscous matrix and, once again, uncouple the velocity components.

Which is not currently explained in the literature, or it has not been sufficiently tested, is that FSM produces an unacceptable variation of the mass (the volume for an incompressible flow) in the vicinity of free-surfaces. This volume variation is typically corrected by modifying the freesurface position in order to preserve the total volume. The correction of the free-surface position just using the total volume value is an unfair algorithm, which only shows the inability of the FSM to preserve the total volume.

The aim of this paper is to explain the reasons for the loss of mass when using the FSM for free-surface flows. In addition, we will present a procedure that overcomes the mass-loss defect when the Navier-Stokes equations for free-surface flows are solved via the FSM using equal-order finite element interpolations for the velocity and the pressure. The efficiency of the mass-loss-free FSM is shown in a number of simple 2D free-surface flow examples solved with linear triangles.
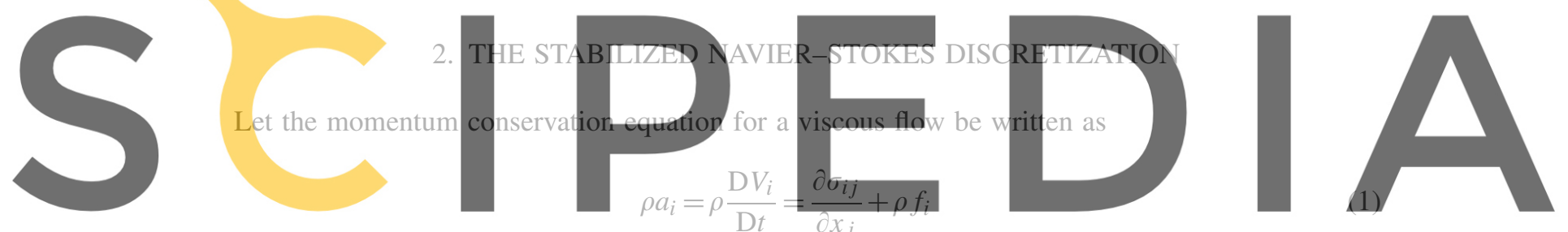

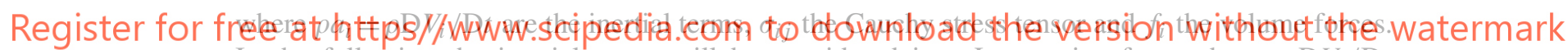
In the following the inertial terms will be considered in a Lagrangian frame, hence, $\mathrm{D} V_{i} / \mathrm{D} t$ denotes the total time derivative. Nevertheless this particular time integration is independent of the problem to be treated in this paper. Thus, the same conclusions are valid for a fluid described in an Eulerian frame.

For a Newtonian flow the following constitutive relations are chosen:

$$
\sigma_{i j}=2 \mu d_{i j}^{\prime}+p \delta_{i j}
$$

where $\mu$ is the viscosity and $d_{i j}^{\prime}$ and $p$ are the deviatory rate of deformation tensor and the pressure defined by

$$
d_{i j}^{\prime}=d_{i j}-\frac{d_{l l}}{3} \delta_{i j}=d_{i j}-\frac{\varepsilon_{V}}{3} \delta_{i j}, \quad d_{i j}=\frac{1}{2}\left(\frac{\partial V_{i}}{\partial x_{j}}+\frac{\partial V_{j}}{\partial x_{i}}\right), \quad p=\operatorname{tr}\left(\frac{\sigma_{i j}}{3}\right)=\frac{\sigma_{l l}}{3}
$$

with the volumetric deformation rate $\varepsilon_{V}=\operatorname{tr}\left(d_{i j}\right)=d_{l l}$.

The boundary conditions are:

$$
\sigma_{i j} v_{j}=\sigma_{n i}=\overline{\sigma_{n i}} \text { on the free-surfaces } \Gamma_{\sigma}
$$

and

$$
V_{i}=\bar{V}_{i} \quad \text { on the remaining boundaries } \Gamma_{V}
$$

where $v_{i}$ are the components of the out-normal vector to the free-surface. 
These equations are completed with the mass conservation equation, which for incompressible flows reads:

$$
\varepsilon_{V}=0
$$

A weighted residual expression for the previous equations is:

$$
\begin{array}{r}
\int_{V} W_{i}\left(\rho \frac{\mathrm{D} V_{i}}{\mathrm{D} t}-\frac{\partial \sigma_{i j}}{\partial x_{j}}-\rho f_{i}\right) \mathrm{d} V+\int_{\Gamma_{\sigma}} W_{i}\left(\sigma_{n i}-\overline{\sigma_{n i}}\right) \mathrm{d} \Gamma=0 \\
\int_{V} W_{p} \varepsilon_{V} \mathrm{~d} V=0
\end{array}
$$

Taking into account the constitutive relations, the weak form may be written as:

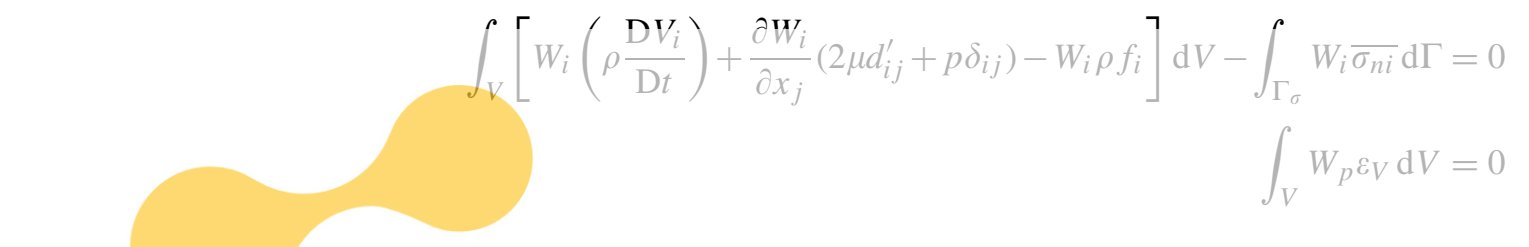

Time integration and spatial discretization: We denote any variable $\alpha$ at time $t_{n}$ as $\alpha^{n}$ and at the current time $t_{n+1}$ as $\alpha^{n+1}$, or simply as $\alpha$. Then the following linear variation for the acceleration will be used for the time integration:
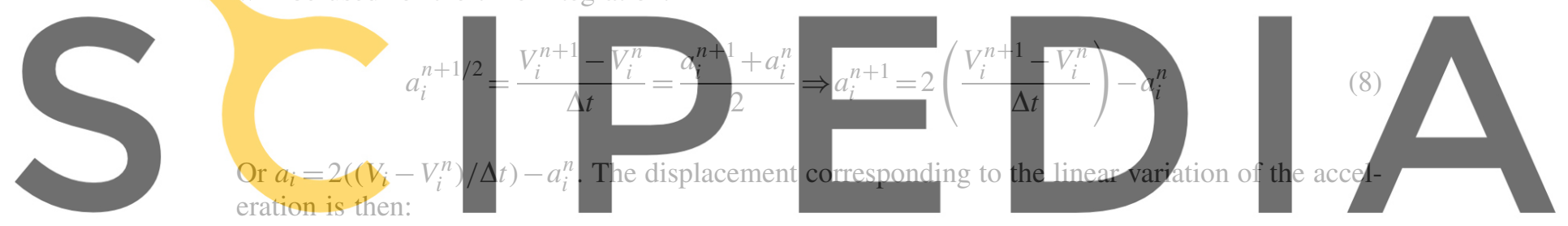

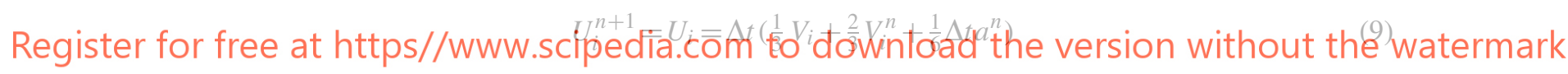

and the updated position of a point is obtained as:

$$
X_{i}^{n+1}=X_{i}=X_{i}^{n}+U_{i}
$$

It must be noted that in the previous expression, $V_{i}^{n+1}$ means $V_{i}^{n+1}\left(X_{i}^{n+1}, t_{n+1}\right)$, i.e. it is the velocity at time $t_{n+1}$ at the position $X_{i}^{n+1}$. This is a particular feature of the Lagrangian formulation [7, 8]. Nevertheless, any other time integration scheme may be used without affecting the conclusions of this paper.

For the spatial integration, the classical FEM linear shape functions will be chosen for both the velocity components and the pressure, i.e.:

$$
\begin{aligned}
V_{i} & =N^{\mathrm{T}} Q_{i} \\
p & =N^{\mathrm{T}} P
\end{aligned}
$$

where $Q_{i}$ and $P$ denote the vectors of local values of the velocity components and the pressure, respectively.

Using a Galerkin method the following system of equations is obtained:

$$
\left\{\frac{2 \rho}{\Delta t}\left[\begin{array}{cc}
M_{i j} & 0 \\
0 & 0
\end{array}\right]+\left[\begin{array}{cc}
K_{i j} & B_{i} \\
B_{j}^{\mathrm{T}} & 0
\end{array}\right]\right\}\left[\begin{array}{c}
Q_{i} \\
P
\end{array}\right]=\left[\begin{array}{c}
G_{i}^{n} \\
0
\end{array}\right]
$$


with:

$$
\begin{aligned}
K_{i j} & =K_{i j}^{1}+K_{i j}^{2}+K_{i j}^{3} \\
K_{i i}^{1} & =\int_{V} \frac{\partial N}{\partial x_{j}}(\mu) \frac{\partial N^{\mathrm{T}}}{\partial x_{j}} \mathrm{~d} V, \quad K_{i j}^{2}=\int_{V} \frac{\partial N}{\partial x_{j}}(\mu) \frac{\partial N^{\mathrm{T}}}{\partial x_{i}} \mathrm{~d} V, \quad K_{i j}^{3}=\int_{V} \frac{\partial N}{\partial x_{i}}\left(-\frac{2 \mu}{3}\right) \frac{\partial N^{\mathrm{T}}}{\partial x_{j}} \mathrm{~d} V \\
M_{i i} & =\int_{V} N N^{\mathrm{T}} \mathrm{d} V, \quad M_{i j}=K_{i j}^{1}=0 \quad \forall i \neq j, \quad B_{i}=\int_{V} \frac{\partial N}{\partial x_{i}} N^{\mathrm{T}} \mathrm{d} V \\
G_{i}^{n} & =\int_{v}\left[N \frac{2 \rho}{\Delta t} V_{i}^{n}+N \rho a_{i}^{n}+N \rho f_{i}\right] \mathrm{d} v+\int_{\Gamma_{\sigma}} N \bar{\sigma}_{n i} \mathrm{~d} \Gamma
\end{aligned}
$$

The boundary conditions for (12) are:

$$
\sigma_{i j} v_{j}=\sigma_{n i}=\overline{\sigma_{n i}} \text { on the free-surfaces } \Gamma_{\sigma}
$$

and

\section{$V_{i}=\bar{V}_{i} \quad$ on the remaining boundaries $\Gamma_{V}$}

FIC stabilization of the pressure terms: It is well known that Equation (12) is unstable for elements with equal-order approximation for velocity and pressure [9] and must be stabilized. A Finite Calculus (FIC) formulation will be used to stabilize the pressure waves [10]. Note however that any other stabilization method may be chosen. The incompressibility equation (5) will be
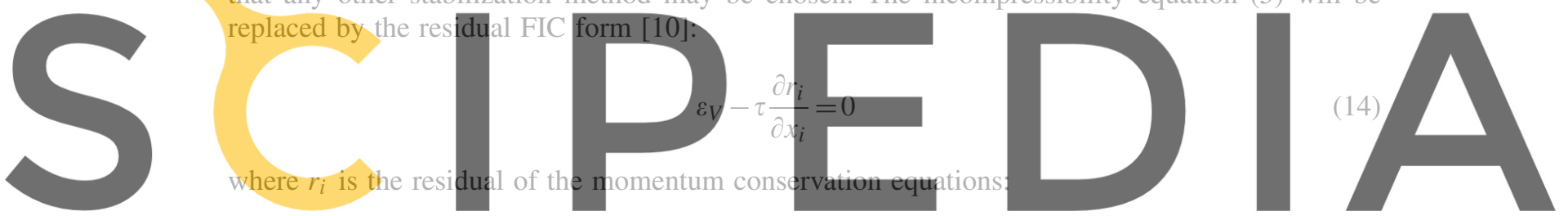

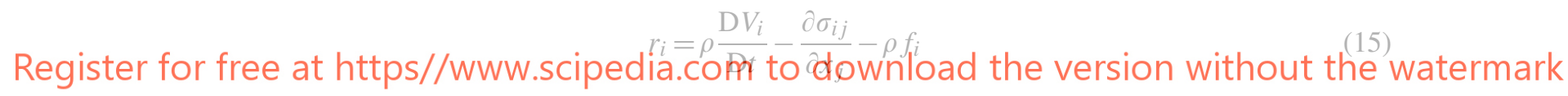

and $\tau$ is a stabilization parameter.

According to the FIC formulation, the stabilization parameter is defined as a constant coefficient for each element with the following value [10]:

$$
\tau=\frac{3 h^{2}}{8 \mu} \quad \text { where } h \text { is a characteristic element length }
$$

The stabilization in Equation (14) is introduced by splitting the residual $r_{i}$ into two parts:

$$
r_{i}=\rho \frac{\mathrm{D} V_{i}}{\mathrm{D} t}-\frac{\partial \sigma_{i j}}{\partial x_{j}}-\rho f_{i}=\rho \frac{\mathrm{D} V_{i}}{\mathrm{D} t}-\rho f_{i}-\frac{\partial}{\partial x_{j}} 2 \mu d_{i j}^{\prime}-\frac{\partial}{\partial x_{i}} p=-\left(\pi_{i}+\frac{\partial}{\partial x_{i}} p\right)
$$

where the $\pi_{i}$ functions represent the projection of the pressure gradients in the velocity field. The use of the $\pi_{i}$ functions ensures the consistency of the stabilization method as the 'full' expression of the residual is retained. The $\pi_{i}$ functions are approximated with the same linear interpolations as for the velocity and the pressure variables:

$$
\pi_{i}=N^{\mathrm{T}} \Pi_{i}
$$

The stabilized weighted equation for the mass conservation becomes:

$$
\int_{V} W_{p}\left(\varepsilon_{V}-\tau \frac{\partial r_{i}}{\partial x_{i}}\right) \mathrm{d} V=\int_{V} W_{p}\left[\varepsilon_{V}+\tau \frac{\partial}{\partial x_{i}}\left(\pi_{i}+\frac{\partial}{\partial x_{i}} p\right)\right] \mathrm{d} V
$$


and the weak form:

$$
\int_{V} W_{p}\left(\varepsilon_{V}-\tau \frac{\partial r_{i}}{\partial x_{i}}\right) \mathrm{d} V=\int_{V} W_{p}\left[\varepsilon_{V} \mathrm{~d} V-\int_{V} \frac{\partial W_{p}}{\partial x_{i}} \tau\left(\pi_{i}+\frac{\partial}{\partial x_{i}} p\right)\right] \mathrm{d} V=0
$$

Computation of the new $\pi_{i}$ variables requires introducing a new set of equations. These are defined by:

$$
\int_{V} W_{i}\left(\pi_{i}+\frac{\partial}{\partial x_{i}} p\right) \mathrm{d} V=0
$$

Equation (21) is a re-statement of the weak form of the momentum equations.

We note that the boundary term appearing from the integration by parts of Equation (20) has been neglected in this work. On the other hand, Equation (21) does not introduce any new boundary condition on the $\pi_{i}$ functions.

The FIC formulation also provides the necessary stabilization for the convective terms appearing in the standard Eulerian formulation. These terms however are not included here as a Lagrangian formulation is used. The discussion on the mass loss in the FSM is independent from accounting or not for the convective terms.

The stabilized form of Navier-Stokes equations after introducing the Galerkin approximation and the corresponding shape functions becomes:
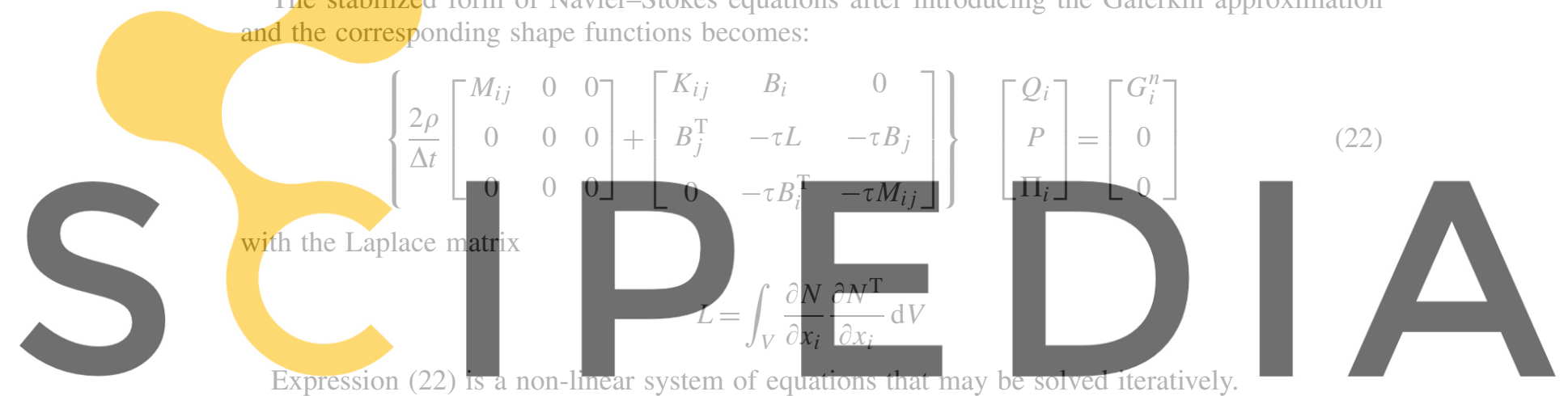

Effectively, system (22) seems to be linear but it is in fact non-linear as it must be solved in the

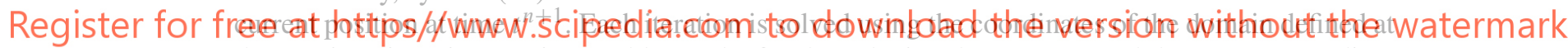

the previous iteration. It gives stable results for the velocity, the pressure and the pressure gradient projections. Of course, the solution of Equation (22) is computational expensive as it involves seven DOFs per node (three velocities, one pressure and three pressure gradient projections).

The problem may be reduced to four DOFs per node by solving the pressure gradient projection unknowns $\Pi_{i}$ as a function of the pressure. From the last line of Equation (22) we obtain:

$$
\Pi_{i}=-M_{i j}^{-1} B_{i}^{\mathrm{T}} P
$$

Then the system to be solved is:

$$
\left\{\frac{2 \rho}{\Delta t}\left[\begin{array}{cc}
M_{i j} & 0 \\
0 & 0
\end{array}\right]+\left[\begin{array}{cc}
K_{i j} & B_{i} \\
B_{j}^{\mathrm{T}} & -\tau\left(L-B_{j} M_{i j}^{-1} B_{i}^{\mathrm{T}}\right)
\end{array}\right]\right\}\left[\begin{array}{c}
Q_{i} \\
P
\end{array}\right]=\left[\begin{array}{c}
G_{i}^{n} \\
0
\end{array}\right]
$$

The evaluation of the matrix $B_{j} M_{i j}^{-1} B_{i}^{\mathrm{T}}$ is simplified by considering a lumped mass matrix. Unfortunately, the bandwidth of that matrix is larger than the original problem (around 5 times larger for 3D problems). This fact makes the problem of solving Equation (23) too expensive.

Fortunately, the pressure gradient projections may be evaluated explicitly from the previous iteration without modifying too much the convergence properties of the non-linear iteration schemes.

Calling $\Pi_{i}^{*}$ the pressure gradient projections at the previous iteration, the algorithm reads as follows:

$$
\left\{\frac{2 \rho}{\Delta t}\left[\begin{array}{cc}
M_{i j} & 0 \\
0 & 0
\end{array}\right]+\left[\begin{array}{cc}
K_{i j} & B_{i} \\
B_{j}^{\mathrm{T}} & -\tau L
\end{array}\right]\right\}\left[\begin{array}{c}
Q_{i} \\
P
\end{array}\right]=\left[\begin{array}{c}
G_{i}^{n} \\
G_{p}^{*}
\end{array}\right]
$$


with

$$
G_{p}^{*}=\tau B_{j} \Pi_{i}^{*}=-\tau B_{j} M_{i j}^{-1} B_{i}^{\mathrm{T}} P^{*}
$$

This algorithm works satisfactorily. It is not necessary to build the total matrix $B_{j} M_{i j}^{-1} B_{i}^{\mathrm{T}}$, it only requires computing vector $G_{p}^{*}$ and the convergence rate is as good as for the previous case (Equation (24)). This will be called in subsequent lines coupled velocity-pressure formulation. The results obtained with this method and the original monolithic scheme will be compared with those of the FSM and the new formulation to be proposed later.

The boundary conditions to solve Equation (25) are exactly as explained previously.

\section{THE FRACTIONAL-STEP METHOD AND THE MASS LOSS ON THE FREE-SURFACE}

The drawback of the equation system represented by Equation (25) is the need to solve simultaneously for the velocity and the pressure unknowns. As stated before, this does not only increase the number of DOFs to be solved simultaneously, but it also turns the system to be ill-conditioned. Hence, large errors using direct solution methods or slow convergence for iterative methods can be expected.

In order to overcome this drawback the FSM as proposed by Chorin $[1,2]$ is used. The FSM consists in splitting the time step solution into two steps. From the first line of Equation (25):
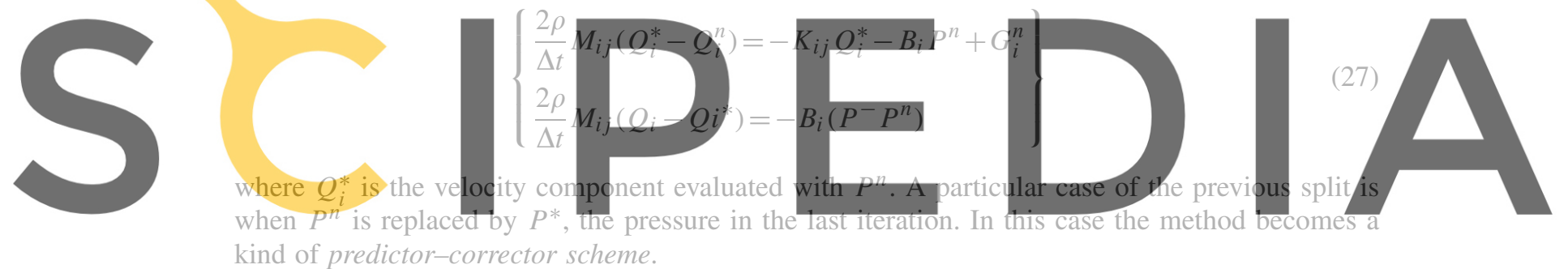

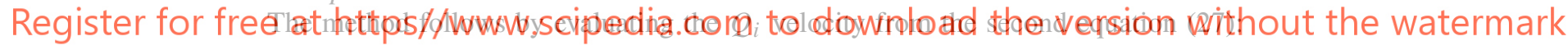

$$
Q_{i}=Q_{i}^{*}-\frac{\Delta t}{2 \rho} M_{i j}^{-1} B_{i}\left(P-P^{n}\right)
$$

Substituting this value into the second equation of (26) gives:

$$
B_{j}^{\mathrm{T}} Q_{i}-\tau L P=B_{j}^{\mathrm{T}}\left[Q_{i}^{*}-\frac{\Delta t}{2 \rho} M_{i j}^{-1} B_{i}\left(P-P^{n}\right)\right]-\tau L P=G_{p}^{*}
$$

Then, the evaluation of the $P$ vector is performed by solving the system:

$$
\left(\frac{\Delta t}{2 \rho} B_{j}^{\mathrm{T}} M_{i j}^{-1} B_{i}+\tau L\right) P=B_{j}^{\mathrm{T}} Q_{i}^{*}+\frac{\Delta t}{2 \rho} B_{j}^{\mathrm{T}} M_{i j}^{-1} B_{i} P^{n}-G_{p}^{*}
$$

Calling,

$$
\hat{L}=B_{j}^{\mathrm{T}} M_{i j}^{-1} B_{i}
$$

the discrete Laplace matrix, Equation (30) becomes:

$$
\left(\frac{\Delta t}{2 \rho} \hat{L}+\tau L\right) P^{=} B_{j}^{\mathrm{T}} Q_{i}^{*}+\frac{\Delta t}{2 \rho} \hat{L} P^{n}-G_{p}^{*}
$$

The new velocity values are evaluated by using the second equation of (27) (classical FSM) or from the first equation (the predictor-corrector method). 
As presented in Equation (29) this FSM has the same drawbacks as the coupled method presented in Equation (25). The bandwidth is too large which makes the solution of Equation (30) too expensive. This procedure will be called FSM with Discrete Laplace matrix (FSMD). (In Reference [11] this method was called Algebraic Splitting Method, but we believe that FSMD is a more proper name.) Remarkably enough, the FSMD method has good properties concerning mass conservation.

The FSD method written in compact form is:

$$
\left\{\frac{2 \rho}{\Delta t}\left[\begin{array}{cc}
M_{i j} & 0 \\
0 & 0
\end{array}\right]+\left[\begin{array}{cc}
K_{i j} & 0 \\
0 & -\left(\tau L+\frac{\Delta t}{2 \rho} \hat{L}\right)
\end{array}\right]\right\}\left[\begin{array}{c}
Q_{i} \\
P
\end{array}\right]=\left[\begin{array}{c}
G_{i}^{n}-B_{i} P^{*} \\
G_{p}^{*}-B_{j}^{\mathrm{T}} Q_{i}^{*}-\frac{\Delta t}{2 \rho} \hat{L} P^{*}
\end{array}\right]
$$

with $P^{*}=P^{n}$ or equal to the pressure of the previous iteration in its predictor-corrector version.

It must be noted that the boundary conditions to solve (33) remain unchanged, this is:

$$
\sigma_{i j} v_{j}=\sigma_{n i}=\overline{\sigma_{n i}} \text { on the free-surfaces } \Gamma_{\sigma}
$$

and

\section{$V_{i}=\bar{V}_{i} \quad$ on the remaining boundaries $\Gamma_{V}$}

In order to avoid the drawback of the large bandwidth, the FSM proposed originally by Chorin $[1,2]$ replaces matrix $B_{i}^{\mathrm{T}} M_{i j}^{-1} B_{j}$ by a Laplace matrix, i.e.:

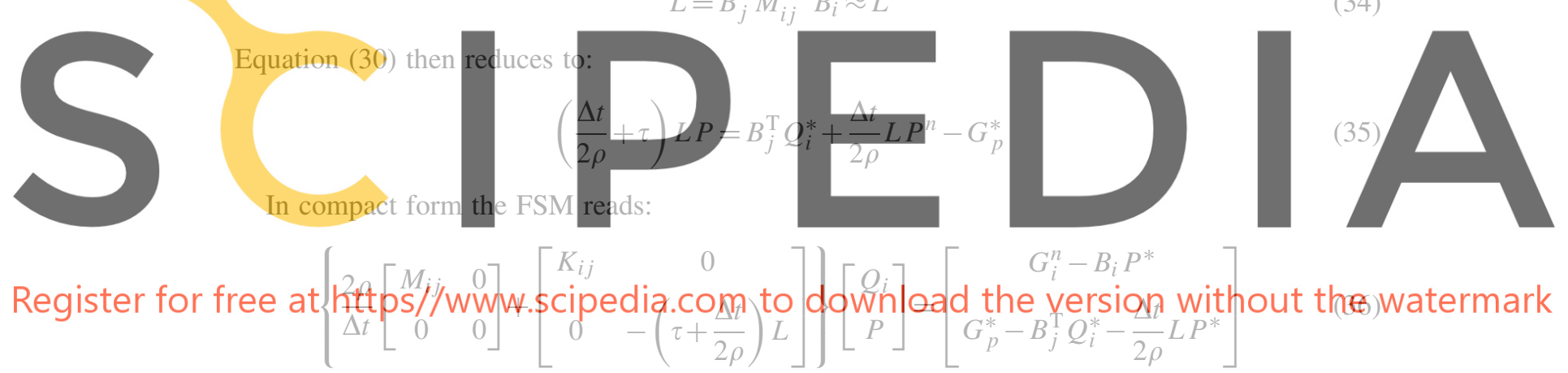

In summary, the FSM involves the following three steps:

Step I:

$$
\text { Evaluate } Q_{i}^{*} \text { from } \frac{2 \rho}{\Delta t} M_{i j}\left(Q_{i}^{*}-Q_{i}^{n}\right)=-K_{i j} Q_{i}^{*}-B_{i} P^{*}+G_{i}^{n}
$$

Step II:

$$
\text { Evaluate } P \text { from }\left(\frac{\Delta t}{2 \rho}+\tau\right) L P=B_{j}^{\mathrm{T}} Q_{i}^{*}+\frac{\Delta t}{2 \rho} L P^{*}-G_{p}^{*}
$$

Step III:

$$
\begin{aligned}
& \text { Evaluate } Q_{i} \text { from } Q_{i}=Q_{i}^{*}-\frac{\Delta t}{2 \rho} M_{i j}^{-1} B_{i}\left(P-P^{*}\right) \\
& \text { or from } \frac{2 \rho}{\Delta t} M_{i j}\left(Q_{i}-Q_{i}^{n}\right)=-K_{i j} Q_{i}^{*}-B_{i} P+G_{i}^{n}
\end{aligned}
$$

This algorithm has been used successfully for many different fluid mechanics problems. As stated before, its computational advantages are so important that it is strongly recommended for as many problems as possible. Unfortunately, it introduces an error in the mass conservation for free-surface flows that makes the results useless. 
Effectively, equation of Step II in all the previous formulations appears from a static condensation of the $\Pi_{i}^{*}$ pressure gradient projection variables. This is an algebraic procedure in which the only approximation is to replace the consistent mass matrix by a lumped one. This algebraic procedure does not affect the global boundary conditions. Now, replacing $B_{j}^{\mathrm{T}} M_{i j}^{-1} B_{i}$ by $L$ is a true transformation of the pressure differential equation and hence, it introduces new boundary conditions. The solution of a Laplace equation requires, for instance, prescribing essential boundary conditions on the pressure and this is not a physical condition.

To overcome this drawback for free-surface flows, it is useful to impose the pressure values on the free-surface. This assumption solves the mathematical problem for solving the Laplace equation, but introduces a spurious boundary condition that affects the volume conservation [12].

The key point: The key problem is that by introducing essential boundary conditions on the pressure terms, the incompressibility condition is relaxed. This can be explained by taking into account that the test functions $W_{p}$ chosen to 'weight' the incompressibility condition are exactly the shape functions $N_{p}$ used to approximate the pressure. Looking at Equation (19) we note that by introducing an essential boundary condition $\left(N_{p}=0\right)$ at some points, we are not forcing the incompressibility condition to be satisfied at that point $\left(W_{p}=0\right)$. This happens at all points where the pressure is prescribed to fixed values and consequently, the corresponding shape function is forced to be zero at those points.

The numerical examples to be shown later will confirm this theoretical aspect of the leakage of mass at the free-surface.

A note concerning the stabilization parameter: In the FSM the stabilization is introduced by the term $(\tau+\Delta t / 2 \rho) L$. For acceptable large time steps, the term $(\Delta t / 2 \rho) L$ introduces the stabilization needed to solve correctly the problem and the $\tau$ parameter may be neglected. For this reason, some
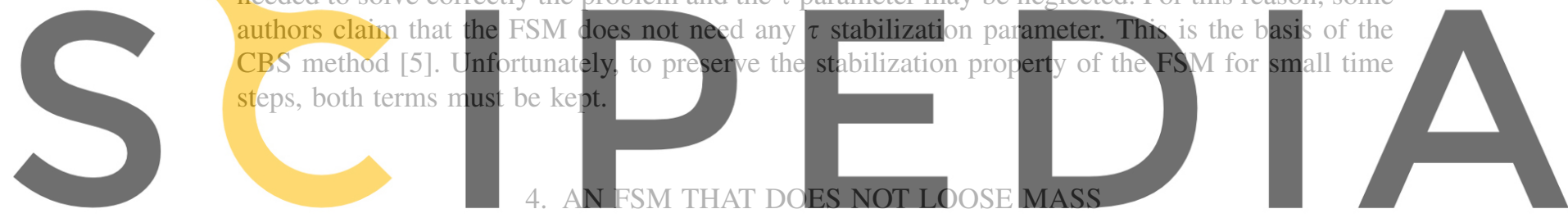

ON THE FREE-SURFACE

Register for free at https//www.scipedia.com to download the version without the watermark

the Laplace matrix. In order to do so without altering the physics of the problem a new term is introduced in the stabilized equation for mass conservation equation (20) as:

$$
\int_{V} W_{p} \varepsilon_{V} \mathrm{~d} V-\int_{\Gamma_{\sigma}} W_{p} \tau_{c}\left(p-p^{*}\right) \mathrm{d} \Gamma-\int_{V}\left[\frac{\partial W_{p}}{\partial x_{i}} \tau\left(\pi_{i}+\frac{\partial}{\partial x_{i}} p\right)\right] \mathrm{d} V=0
$$

The new free-surface boundary term in the second integral of Equation (37) avoids the singularity of the Laplace form and eliminates the need for imposing essential boundary conditions to solve the pressure equation.

There are several ways to justify the free-surface boundary term in Equation (37) and to evaluate the parameter $\tau_{c}$. One is the following:

The total domain is split into two parts, a fully incompressible domain $V_{0}$, with $\varepsilon_{V}=0$ and a second domain $V_{\kappa}$, near the free-surface, with a compressibility coefficient such that:

$$
\varepsilon_{V}=\frac{1}{\kappa} \frac{\mathrm{D} p}{\mathrm{D} \theta}
$$

with $\kappa=\rho C^{2}$ the bulk compressibility coefficient, being $C$ a fictitious speed of sound and $\theta$ any time variation.

With this assumption, Equation (20) becomes:

$$
\int_{V_{0}} W_{p} \varepsilon_{V} \mathrm{~d} V+\int_{V_{k}} W_{p}\left[\varepsilon_{V}-\frac{1}{\kappa} \frac{\mathrm{D} p}{\mathrm{D} \theta}\right] \mathrm{d} V-\int_{V}\left[\frac{\partial W_{p}}{\partial x_{i}} \tau\left(\pi_{i}+\frac{\partial}{\partial x_{i}} p\right)\right] \mathrm{d} V=0
$$




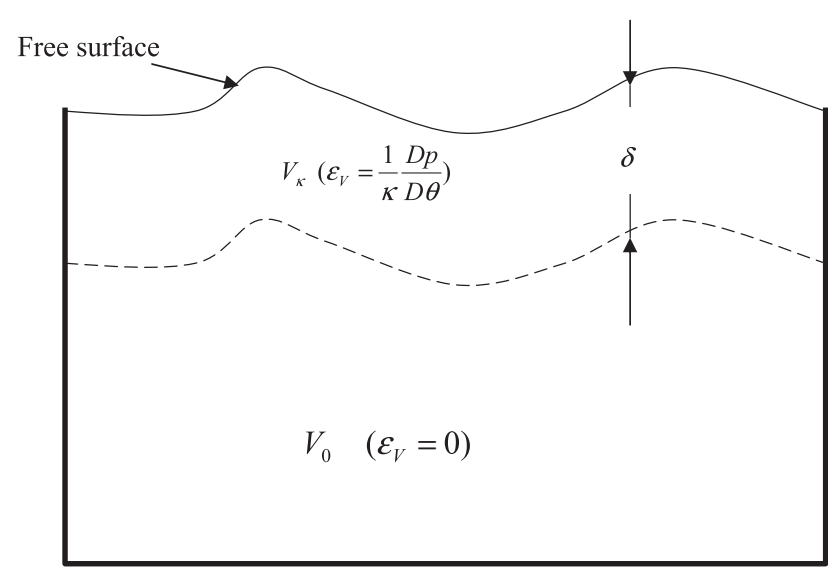

Figure 1. Split of the volume into two parts, a compressible and an incompressible one.

Supposing that the domain $V_{\kappa}$ is a thin layer of thickness $\delta$ around the free-surface (see Figure 1), Equation (39) may be written as:

$$
\int_{V} W_{p} \varepsilon_{V} \mathrm{~d} V-\delta \int_{\Gamma_{\sigma}} W_{p} \frac{1}{\kappa} \frac{\mathrm{D} p}{\mathrm{D} \theta} \mathrm{d} V-\int_{V}\left[\frac{\partial W_{p}}{\partial x_{i}} \tau\left(\pi_{i}+\frac{\partial}{\partial x_{i}} p\right)\right] \mathrm{d} V=0
$$

The thickness $\delta$ may change within the iterations, decreasing its value until it becomes zero

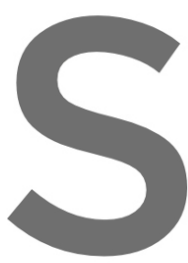
when convergence is achieved.

For instance, approximatin

Equation (40) reads:
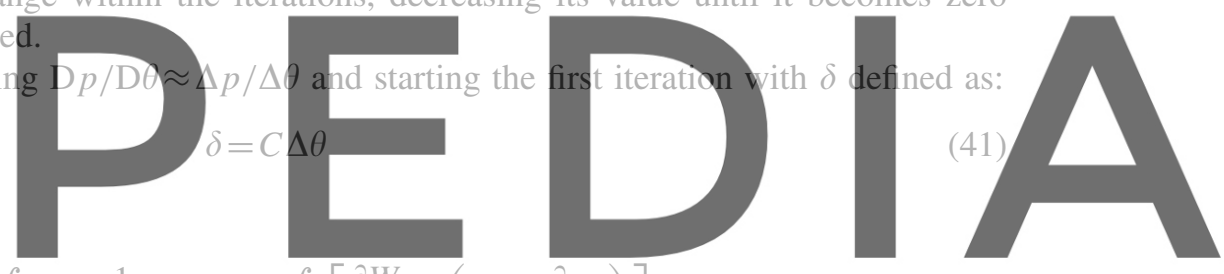

$$
\int W_{p} \varepsilon_{V} \mathrm{~d} V-\int W_{p} \frac{1}{p C} \Delta p \mathrm{~d} V-\int\left[\frac{\partial W_{p}}{\partial x_{i}} \tau\left(\pi_{i}+\frac{\partial}{\partial x_{i}} p\right)\right] \mathrm{d} V=0
$$

Register for free at http's//www.scipedia.com to dowhload the version without the watermark which justifies Equation (37).

The Galerkin discretization process for Equation (42) leads to:

$$
-\left(\tau L+\tau_{c} M_{c}\right) P=-B_{j}^{\mathrm{T}} Q_{i}+G_{p}^{*}-\tau_{c} M_{c} P^{*}
$$

with

$$
\tau_{c}=\frac{1}{\rho C}
$$

and

$$
M_{c}=\int_{\Gamma_{\sigma}} N N^{\mathrm{T}} \mathrm{d} \Gamma
$$

Introducing Equation (43) into the velocity-pressure system Equation (36) reads:

$$
\begin{gathered}
\left\{\frac{2 \rho}{\Delta t}\left[\begin{array}{cc}
M_{i j} & 0 \\
0 & 0
\end{array}\right]+\left[\begin{array}{cc}
K_{i j} & 0 \\
0 & -\left(\tau+\frac{\Delta t}{2 \rho}\right) L-\tau_{c} M_{c}
\end{array}\right]\right\}\left[\begin{array}{c}
Q_{i} \\
P
\end{array}\right] \\
=\left[\begin{array}{c}
G_{i}^{n}-B_{i} P^{*} \\
G_{p}^{*}-B_{j}^{\mathrm{T}} Q_{i}^{*}-\frac{\Delta t}{2 \rho} L P^{*}-\tau_{c} M_{c} P^{*}
\end{array}\right]
\end{gathered}
$$


This method will be called FSM with Incompressibility on the free-surface (FSMI).

The fictitious speed of sound $C$ must be chosen so as to avoid the singularity of the Laplace matrix during the iterations. A large value of $C$ means that the new stabilization matrix $\tau_{c} M_{c}$ is nearly zero and then, the Laplace equation remains singular. On the other hand, a small value of $C$ defines a large compressible region, and this can only be eliminated after many iterations. Nevertheless the value introduced for $C$ does not affect the results, as the thickness $\delta$ of the compressible region becomes zero in the convergence limit and the total domain becomes incompressible. A practical value for $C$ will be discussed in the examples.

The new free-surface boundary term avoids the singularity of the Laplace matrix. The pressure equation may be solved accurately without introducing any essential boundary condition. Owing to these reasons, the incompressibility of the domain is fully preserved.

We note however that the new term $\tau_{c} M_{c}$ may also be added in all previous formulations. It is however unnecessary in all formulations that do not require solving a Laplace equation for the pressure. Nevertheless, it may be interesting to add this term in the FSMD (Equation 33). For small time stens, this formulation reduces to a Laplace form, in spite of using a non-zero $\tau$ parameter.

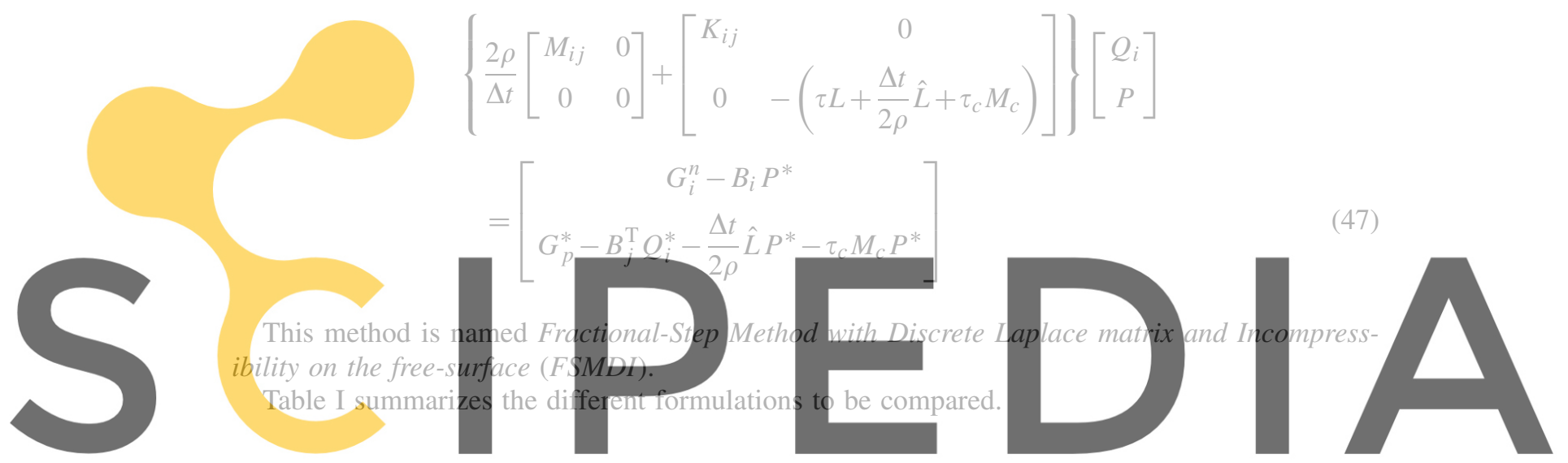

\section{Register for free at https//www.scipedia.com to download the version without the watermark}

The first example was designed in order to favour the volume loss in an FSM. Effectively, freesurface flows with small waves where the curvature is large are examples where the possibility that the essential boundary conditions needed in the FSM affect more the incompressibility of the fluid near the free-surface.

The example presented is a $2 \mathrm{D}$ viscous flow in a recipient with 5 initial waves on the freesurface. A very coarse mesh of $20 \times 20$ triangular elements was used as shown in Figure 2. Slipping conditions on the lateral walls and on the bottom of the recipient were considered.

In spite of its simplicity this example is a very difficult test for free-surface problems and it is proposed here as a test for this kind of fluid mechanics problems.

The physical and geometrical properties are: $\mu=0.01(\mathrm{~kg} / \mathrm{cms}), \rho=0.001\left(\mathrm{~kg} / \mathrm{cm}^{3}\right), g=$ $981\left(\mathrm{~cm} / \mathrm{s}^{2}\right)$. The sides of the container are $L=100(\mathrm{~cm}), H=100(\mathrm{~cm})$ and the initial wave height is $10(\mathrm{~cm})$.

The different methods described in the paper have been evaluated with this simple example (but very difficult one from the mass losing point of view). In all cases the non-linear iterations were performed until the norm $L^{2}$ of the displacement field divided by the length of the recipient become smaller than $10^{-06}$. The stabilization parameter was evaluated by Equation (18) using $h=10$. For all methods, the mesh was fixed to the initial one, changing for each iteration (and/or time step) the coordinates of the nodes only. When the mesh becomes too distorted the algorithm was stopped.

Several other examples have been proved to check the efficiency of the algorithm proposed concerning simplicity and mass conservation. Two more complicated examples are shown. One is the sloshing problem with small waves and other is a problem concerning the mixing of two 
Table I. Schematic comparison of different algorithms to be numerically compared.

Coupled velocity-pressure-gradient projection formulation $(V-P-\Pi)$ :

$$
\left\{\frac{2 \rho}{\Delta t}\left[\begin{array}{cc}
M_{i j} & 0 \\
0 & 0
\end{array}\right]+\left[\begin{array}{cc}
K_{i j} & B_{i} \\
B_{j}^{\mathrm{T}} & -\left(\tau L+B_{j} M_{i j}^{-1} B_{i}^{\mathrm{T}}\right)
\end{array}\right]\right\}\left[\begin{array}{c}
Q_{i} \\
P
\end{array}\right]=\left[\begin{array}{c}
G_{i}^{n} \\
0
\end{array}\right]
$$

Coupled velocity-pressure formulation $(V-P)$ :

$$
\left\{\frac{2 \rho}{\Delta t}\left[\begin{array}{cc}
M_{i j} & 0 \\
0 & 0
\end{array}\right]+\left[\begin{array}{cc}
K_{i j} & B_{i} \\
B_{j}^{\mathrm{T}} & -\tau L
\end{array}\right]\right\}\left[\begin{array}{c}
Q_{i} \\
P
\end{array}\right]=\left[\begin{array}{c}
G_{i}^{n} \\
G_{p}^{*}
\end{array}\right]
$$

Fractional-step method with discrete laplace matrix (FSMD):

$$
\left\{\frac{2 \rho}{\Delta t}\left[\begin{array}{cc}
M_{i j} & 0 \\
0 & 0
\end{array}\right]+\left[\begin{array}{cc}
K_{i j} & 0 \\
0 & -\left(\tau L+\frac{\Delta t}{2 \rho} \hat{L}\right)
\end{array}\right]\right\}\left[\begin{array}{c}
Q_{i} \\
P
\end{array}\right]=\left[\begin{array}{c}
G_{i}^{n}-B_{i} P^{*} \\
G_{p}^{*}-B_{j}^{\mathrm{T}} Q_{i}^{*}-\frac{\Delta t}{2 \rho} \hat{L} P^{*}
\end{array}\right]
$$

Fractional-step method (FSM):

$$
\left\{\frac{2 \rho}{\Delta t}\left[\begin{array}{cc}
M_{i j} & 0 \\
0 & 0
\end{array}\right]+\left[\begin{array}{cc}
K_{i j} & 0 \\
0 & -\left(\tau+\frac{\Delta t}{2 \rho}\right) L
\end{array}\right]\right\}\left[\begin{array}{c}
Q_{i} \\
P
\end{array}\right]=\left[\begin{array}{c}
G_{i}^{n}-B_{i} P^{*} \\
G_{p}^{*}-B_{j}^{\mathrm{T}} Q_{i}^{*}-\frac{\Delta t}{2 \rho} L P^{*}
\end{array}\right]
$$

Fractional-step method with incompressibility on the free-surface (FSMI):

$$
\left\{\frac{2 \rho}{\Delta t}\left[\begin{array}{cc}
M_{i j} & 0 \\
0 & 0
\end{array}\right]+\left[\begin{array}{cc}
K_{i j} & 0 \\
0 & -\left(\tau+\frac{\Delta t}{2 \rho}\right) L-\tau_{c} M_{c}
\end{array}\right]\right\}\left[\begin{array}{c}
Q_{i} \\
P
\end{array}\right]=\left[\begin{array}{c}
G_{i}^{n}-B_{i} P^{*} \\
G_{p}^{*}-B_{j}^{\mathrm{T}} Q_{i}^{*}-\frac{\Delta t}{2 \rho} L P^{*}-\tau_{c} M_{c} P^{*}
\end{array}\right]
$$

Fractional-step method with discrete laplace matrix and incompressibility on the free-surface (FSMDI):

$$
\left\{\frac{2 \rho}{\Delta t}\left[\begin{array}{cc}
M_{i j} & 0 \\
0 & 0
\end{array}\right]+\left[\begin{array}{cc}
K_{i j} & 0 \\
0 & -\left(\tau L+\frac{\Delta t}{2 \rho} \hat{L}+\tau_{c} M_{c}\right)
\end{array}\right]\right\}\left[\begin{array}{c}
Q_{i} \\
P
\end{array}\right]=\left[\begin{array}{c}
G_{i}^{n}-B_{i} P^{*} \\
G_{p}^{*}-B_{j}^{\mathrm{T}} Q_{i}^{*}-\frac{\Delta t}{2 \rho} \hat{L} P^{*}-\tau_{c} M_{c} P^{*}
\end{array}\right]
$$

fluid flows with different densities. It must be noted that the volume loss for the FSM is not as spectacular in these examples as in the previous one, but adding the free-surface stabilization matrix proposed here improves considerably the results.

\subsection{Coupled velocity-pressure formulation $(V-P)$}

Figure 3 shows the point positions for different time steps using the coupled velocity pressure (V-P). No differences were found in the results using the (V-P-П) formulation. For this reason, these results are considered as 'exact'. A time step $\Delta t=10^{-2}$ was used. Figure 3 shows that there is a natural frequency of the waves of around $0.30 \mathrm{~s}$. The waves are dumped due to the viscosity effect until they disappear after $0.75 \mathrm{~s}$.

The variation of volume at each time step was evaluated as a function of the original volume. This is represented in percentage value in Figure 4. We observe that the volume variation is practically negligible in the V-P formulation, reading a maximum value of $10^{-4}$ at $t=0.16 \mathrm{~s}$.

The maximum number of iterations performed to reach the norm $10^{-06}$ was equal to 3 and the average number of iterations during the entire time evaluation was 2.28. It must be noted that the iterations were performed on the total velocity-pressure coupled system. This means that despite the small number of iterations, each one is more expensive than the fractional-step iteration. 


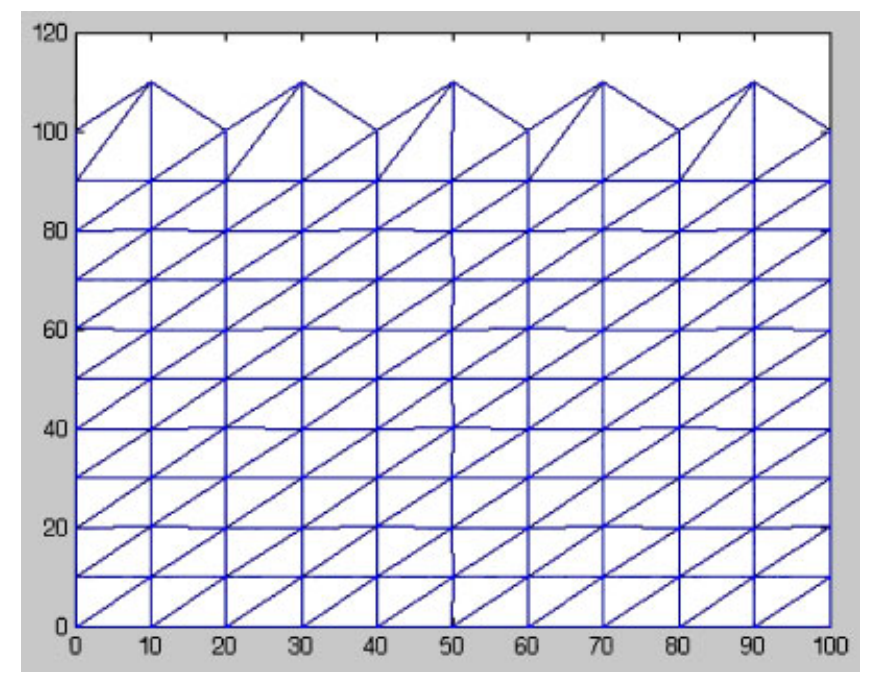

Figure 2. Mesh and initial geometry.

\subsection{Fractional-Step method (FSM)}

The same problem was solved using the classical FSM with identical geometrical and physical parameters. The only difference, as explained before, is that in the FSM method essential boundary conditions for the pressure variable must be imposed on the free-surface. For this problem the value of pressure was set equal to zero at the free-surface nodes.

Figure 5 shows the position of the points after $0.1 \mathrm{~s}$. The process was stopped after this time because the mesh becomes too distorted and a remeshing process was needed. We note that remeshing was not performed in these examples.

Figure 5 shows also the percentage of volume variation. After $0.1 \mathrm{~s}$ the volume variation is near $4 \%$. Observing carefully the point positions in Figure 5 we can conclude that the main volume loss is near the free-surface. This is in agreement with that explained in the paper.

\subsection{FSM with Incompressibility on the free-surface (FSMI)}

The same problem may be solved accurately by adding the boundary matrix $\tau_{c} M_{c}$ as described in Section 4 (FSMI method). The parameter $\tau_{c}=1 / \rho C$ was fixed using $C=100(\mathrm{~cm} / \mathrm{s})$. Smaller parameters $(C=10$ and 1$)$ were also tested with identical results but with a double number of iterations by time step. Larger values of $C$ do not avoid the singularity of the Laplace matrix. We understand that the larger values of $C$ that may be used depend in a great extent to the precision of the computer used. In fact, the $C$ parameter must be adjusted in order to provide the Laplace matrix the proper rank so as to eliminate singularity.

Figure 6 shows the position of the point at different time steps. The results are in agreement with those obtained with the coupled velocity-pressure formulation, with the same frequency and the same time to reach a smooth solution.

Figure 7 shows the percentage of volume variation with a maximum value of $2 \times 10^{-3}$.

The maximum number of iterations was equal to 4 and the average in the total time was 2.5 . Note that this method solves for the velocity and pressure in an uncoupled manner as in the standard FSM. The bandwidth of the matrices is of the same order. For small values of the viscosity each velocity component can be solved in an uncoupled manner.

The method was also tested for different time steps without any difficulty. Very small time steps do not produce any instability in the results. Large time steps introduce the classical numerical error expected for this kind of integration scheme.

In order to check the validity of $\tau$ and $\tau_{c}$ parameters the same problem was solved for a very small viscosity value $\mu=10^{-6}$. The solution was stopped after $0.1 \mathrm{~s}$ due to the distortion of the 


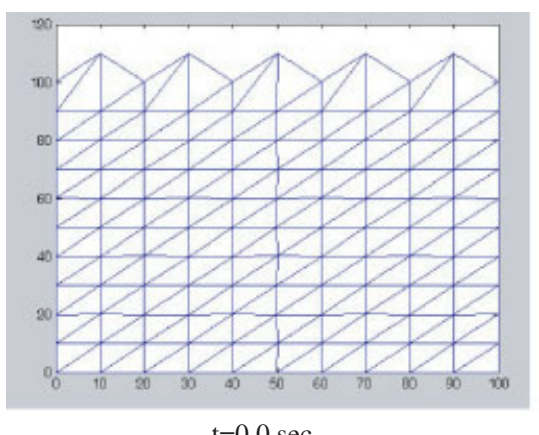

$\mathrm{t}=0.0 \mathrm{sec}$.

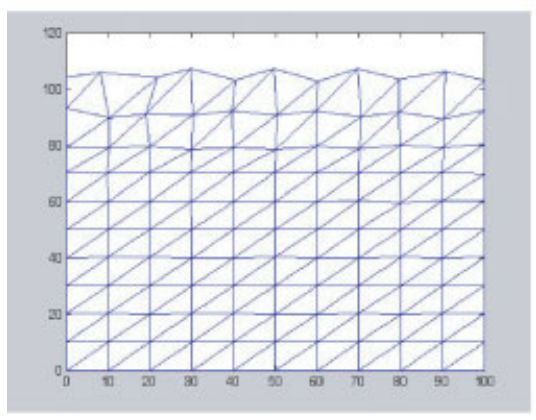

$\mathrm{t}=0.30 \mathrm{sec}$.

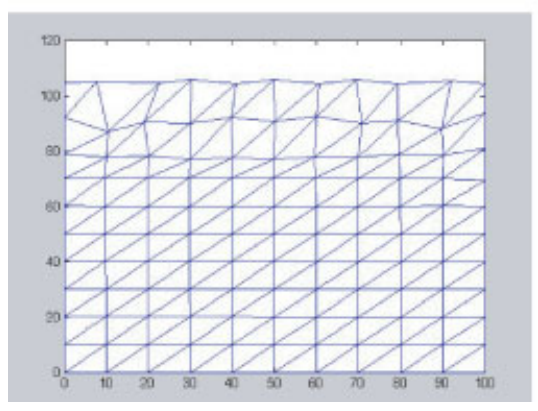

$\mathrm{t}=0.60 \mathrm{sec}$

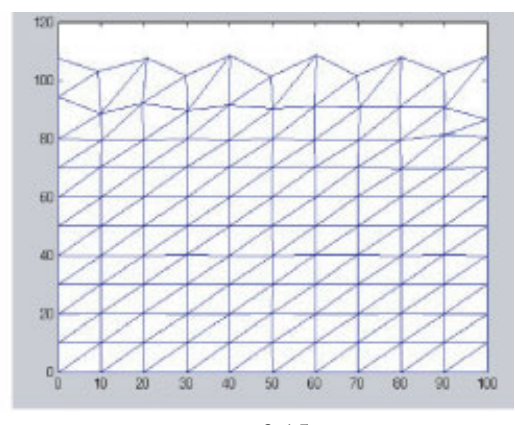

$\mathrm{t}=0.15 \mathrm{sec}$.

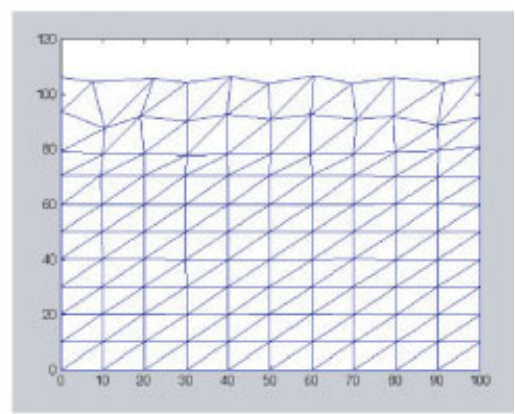

$\mathrm{t}=0.45 \mathrm{sec}$.

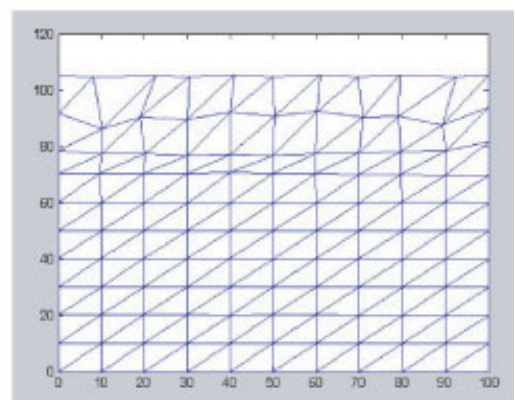

$\mathrm{t}=0.75 \mathrm{sec}$.

Figure 3. Different time steps for the V-P formulation.

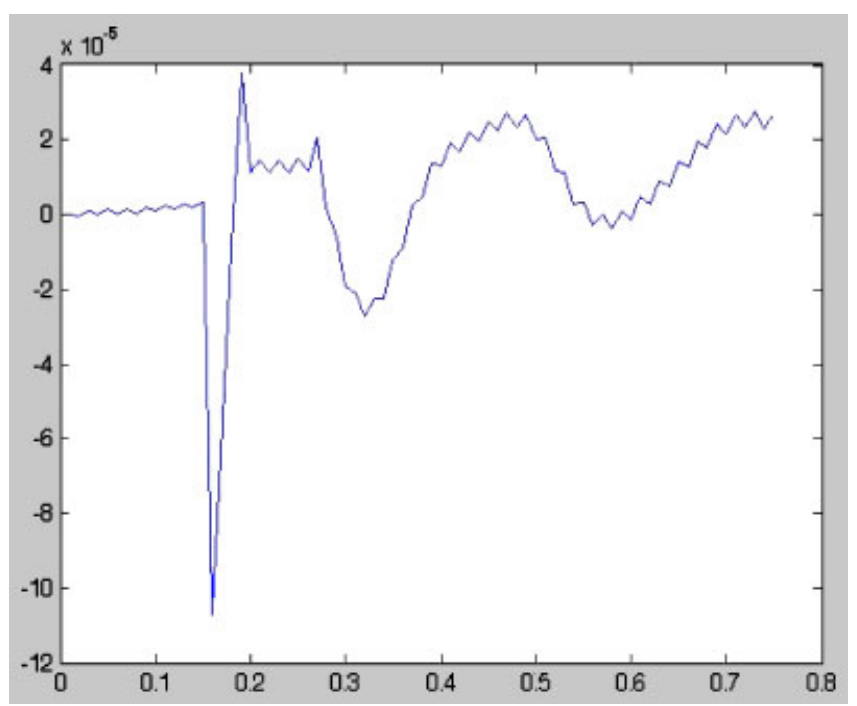

Figure 4. V-P formulation: \% of the volume variation at different time steps. 

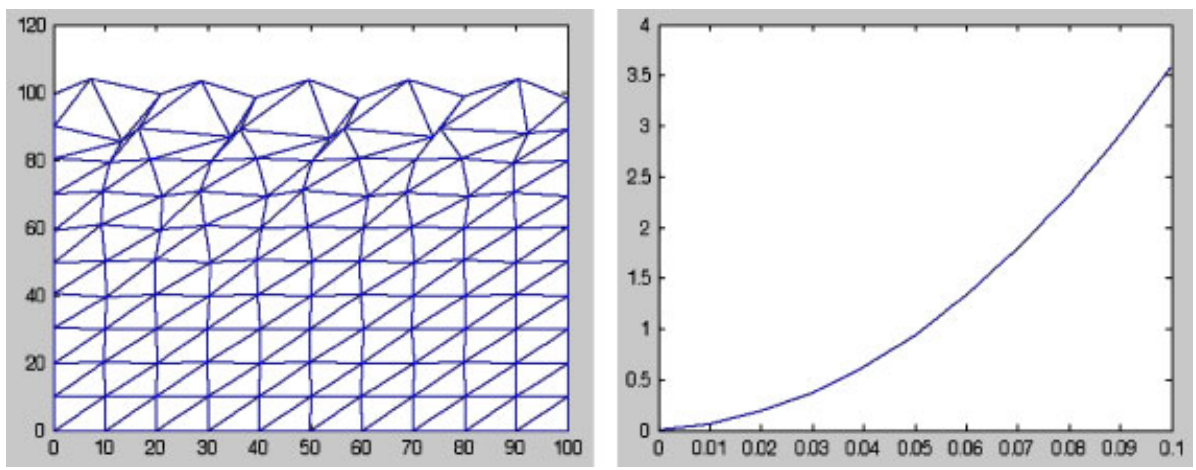

Figure 5. Fractional step method. Left: positions of the points after $0.10 \mathrm{~s}$. Right: $\%$ of volume variation at different time steps.

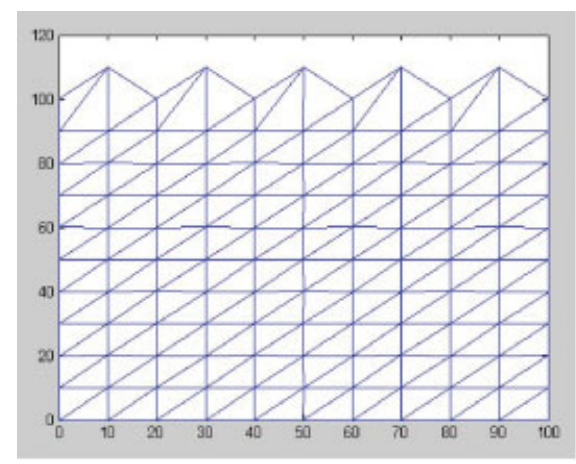

$\mathrm{t}=0.0 \mathrm{sec}$.

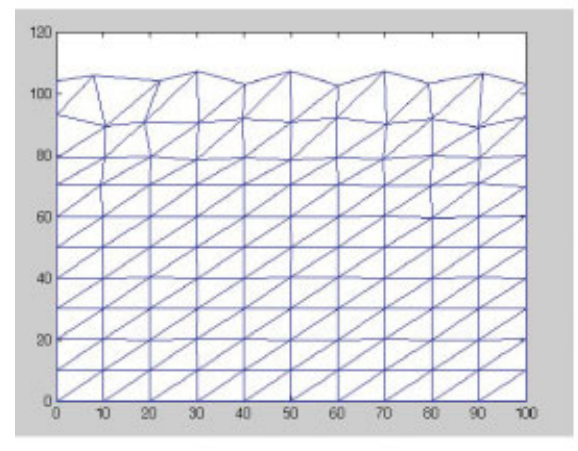

$\mathrm{t}=0.30 \mathrm{sec}$.

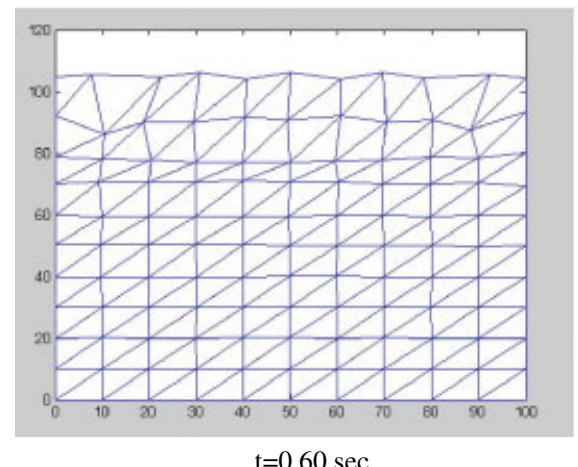

$\mathrm{t}=0.60 \mathrm{sec}$
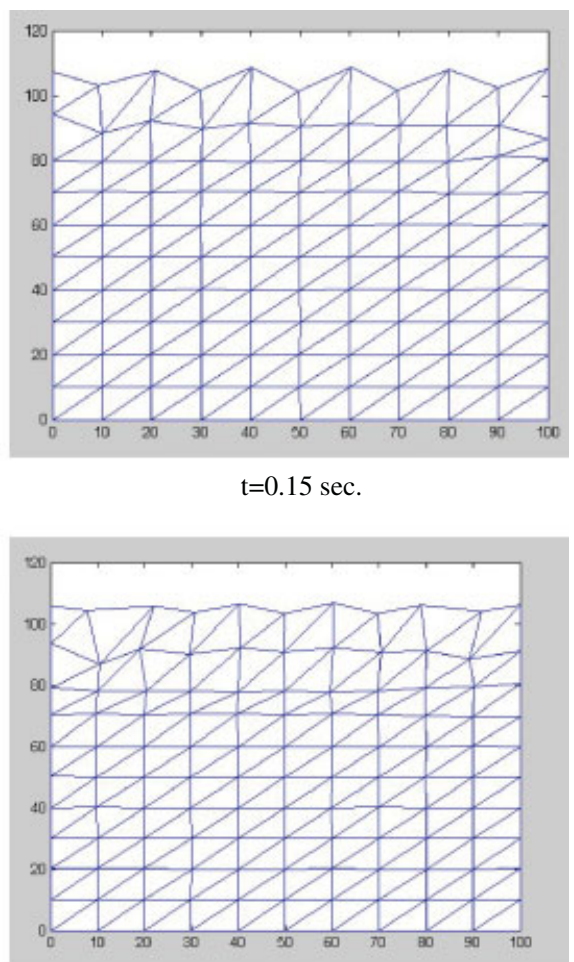

$\mathrm{t}=0.45 \mathrm{sec}$.

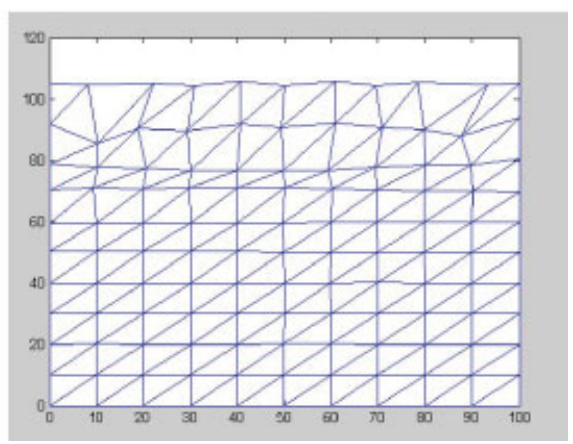

$\mathrm{t}=0.75 \mathrm{sec}$.

Figure 6. Different time steps for the FSMI formulation. 


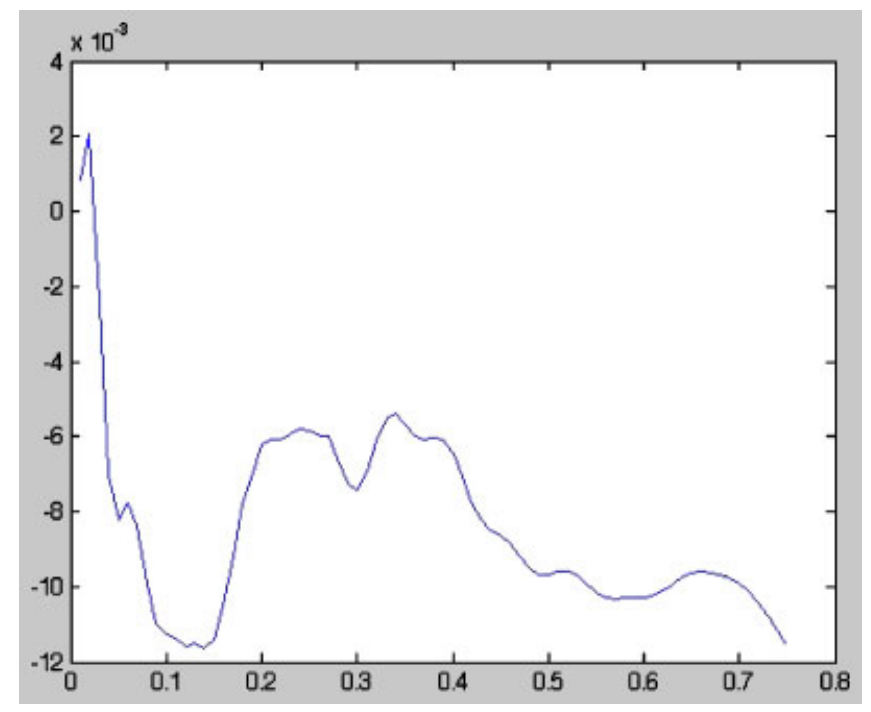

Figure 7. FSMI formulation: $\%$ of the volume variation at different time steps.

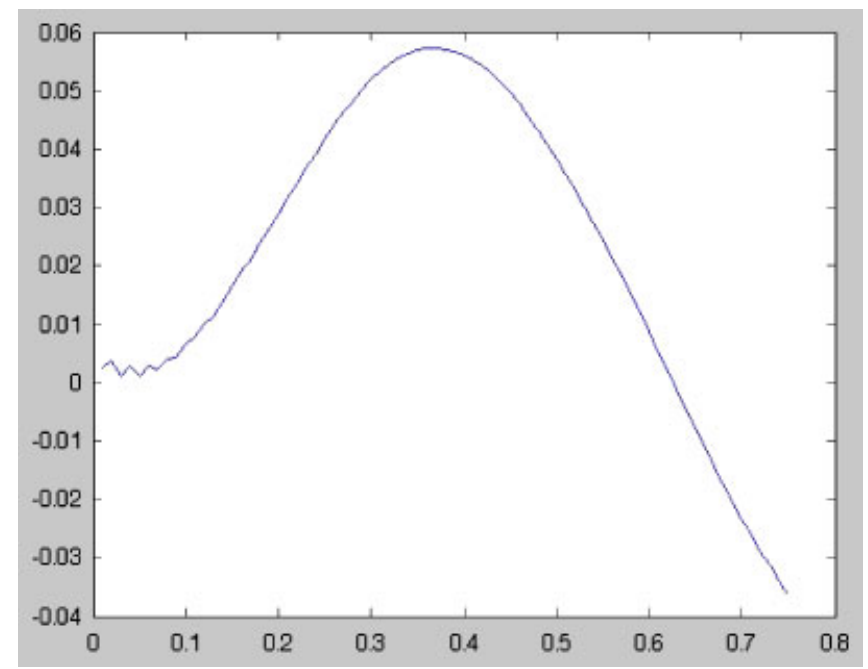

Figure 8. FSMD formulation: \% of the volume variation at different time steps.

mesh. Even though, the pressure and the percentage of volume variation were in agreement with the results obtained with the V-P formulation.

\subsection{FSM with Discrete Laplace matrix (DFS)}

Finally, the FSMD method was also tested. The results for the point coordinates and the frequency of the waves are in good agreement with those obtained with the V-P and FSMI formulations. The percentage of the volume change is shown in Figure 8. The maximum number of iterations was equal to 8 with an average of 2.56 iterations by time step. We note that in the FSMD formulation, the pressure is decoupled from the velocity but the pressure matrix has a large bandwidth. This is probably the reason why the number of iterations needed to converge to the same error is larger. Note also that each iteration is considerably more expensive than in the FSMI formulation due to the larger bandwidth (five times larger than the front of the Laplace matrix for a standard 3D problem). Nevertheless, the variation of the volume rate is acceptable, as claimed in Reference [11] with a maximum of $0.06 \%$ at $0.4 \mathrm{~s}$ in this example. 


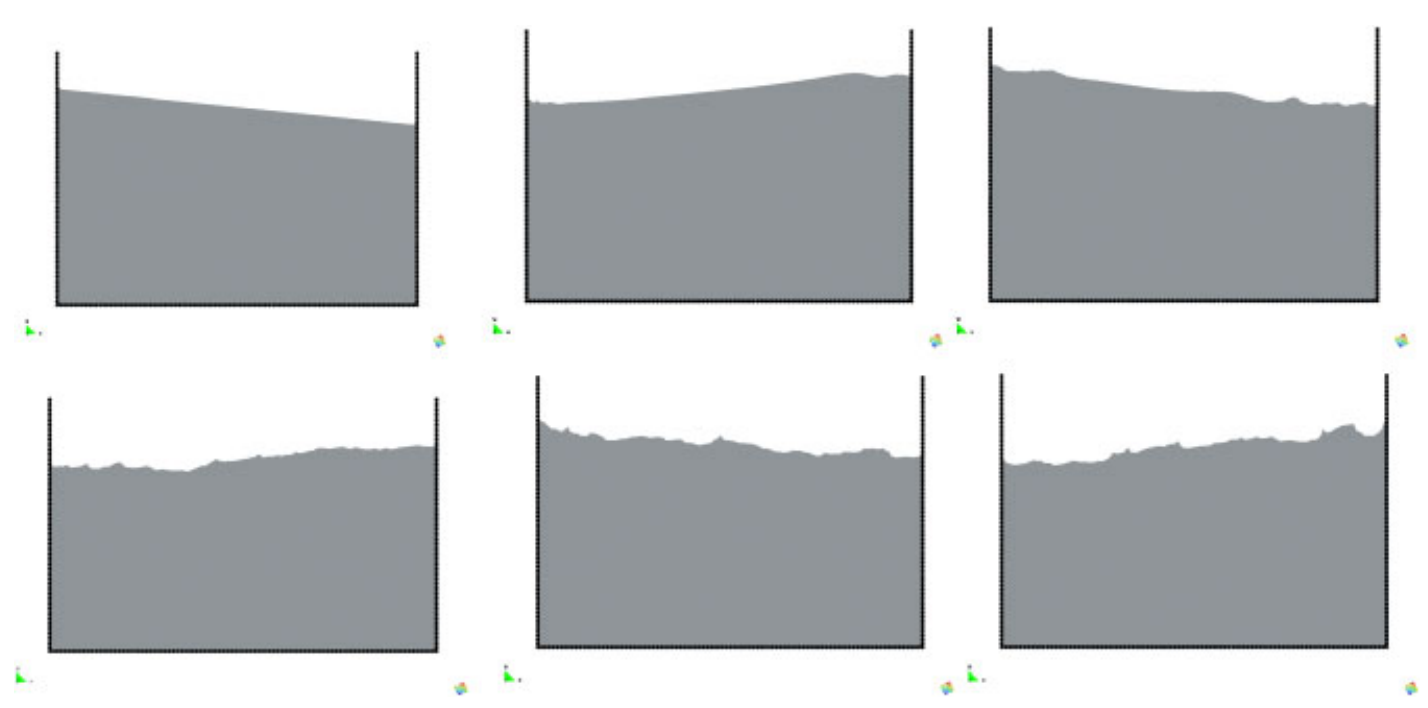

Figure 9. Sloshing test. Free-surface positions at different time steps.

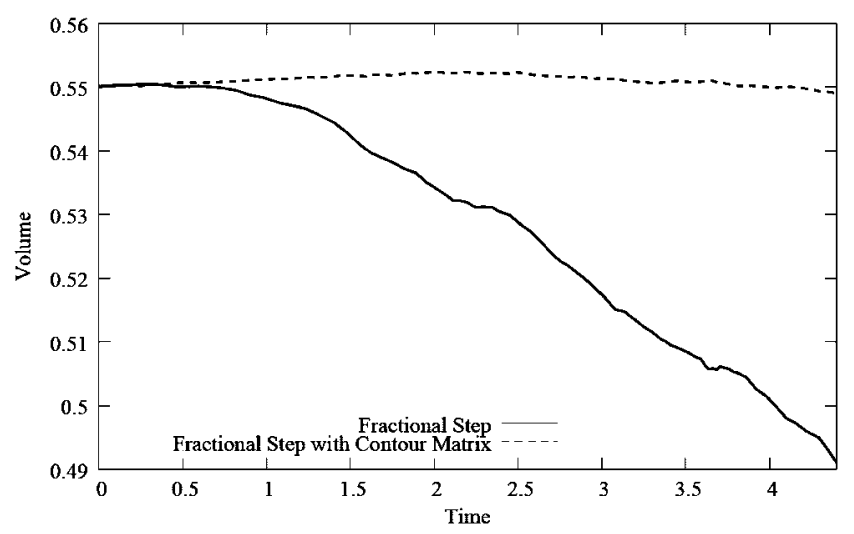

Figure 10. Sloshing test. Total volume variation in standard fractional-step method (FSM) and including the proposed Contour Matrix (FSMI).

\subsection{Sloshing with small free-surface waves}

A classical sloshing problem has been solved to test also the volume variation of the different methods. The initial position of the fluid is represented in Figure 9 together with position of the fluid at different time steps. A rectangular domain of fluid of $1 \mathrm{~m}$ wide with a free-surface is initially disturbed from the equilibrium position with a deep of $0.6 \mathrm{~ms}$ in the left and $0.5 \mathrm{~ms}$ in the right. The density and viscosity of the fluid are $1000 \mathrm{~kg} / \mathrm{m}^{3}$ and $0.01 \mathrm{Pas}$, respectively. The rectilinear sloped free-surface of the fluid was chosen in order to excite the small waves. Effectively, classical sloshing tests start from a sinusoidal free-surface without small waves on the free-surface. We are particularly interested in small free-surface waves because the mass-loss process increases when the free-surface has many broken curves.

Figure 10 shows the fluid volume at different time steps using the standards FSM and the FSMI using the new boundary mass matrix. We can see that the FSMI method does not show any important volume variation while the FSM leads to catastrophic results.

\subsection{Mixing two fluids with different densities}

A rectangular recipient $1 \mathrm{~m}$ wide and $0.5 \mathrm{~m}$ deep was filled up with two different fluids: one laying at the bottom part of the recipient with a density of $2300 \mathrm{~kg} / \mathrm{m}^{3}$ and the other one floating at the 


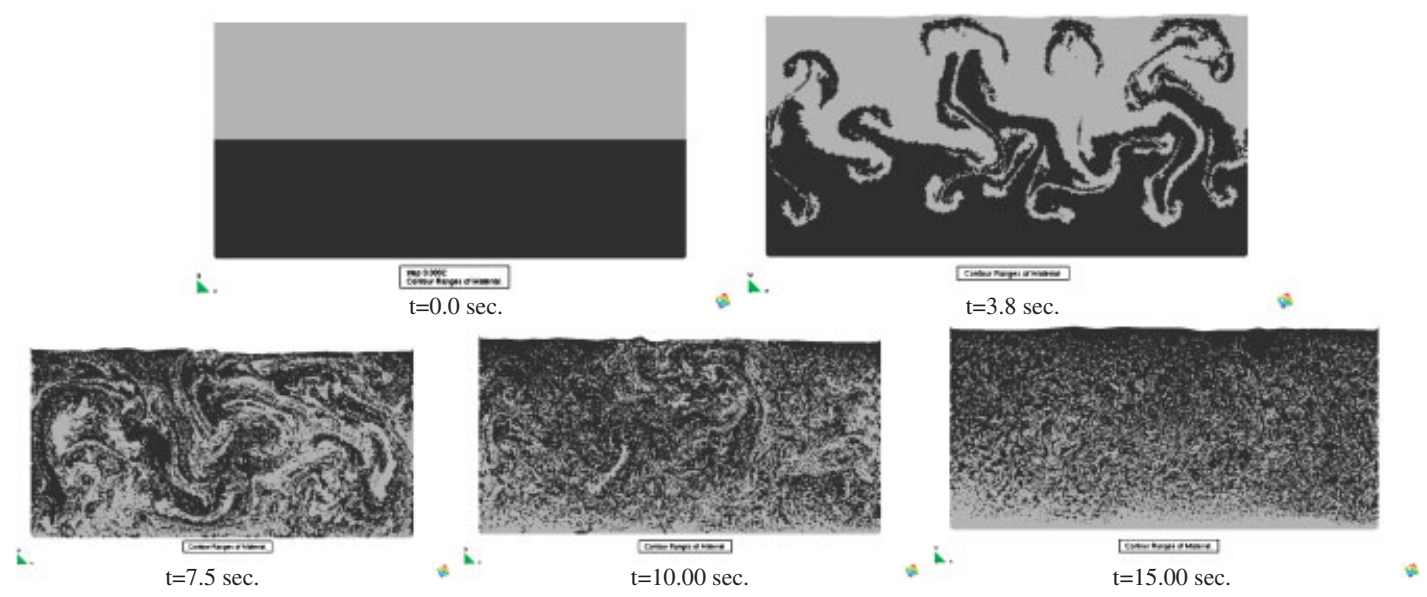

Figure 11. Mixing two fluids with different densities.

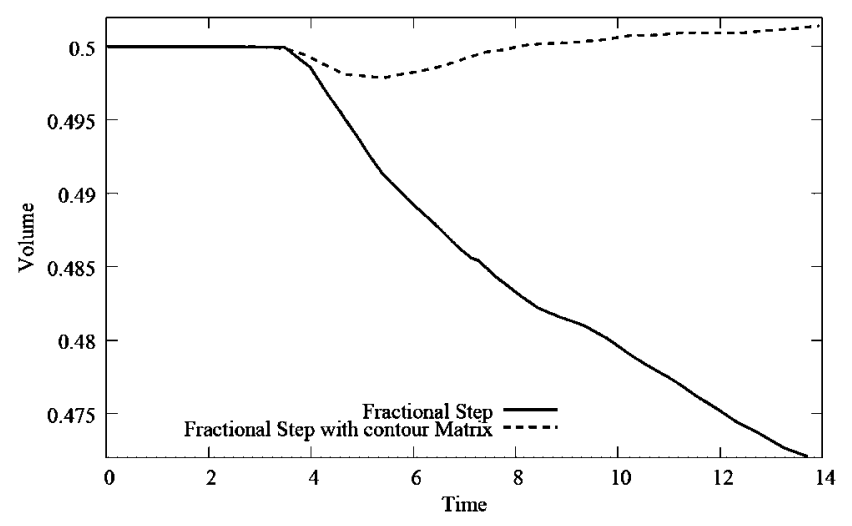

Figure 12. Total volume variation in standard fractional-step method, FSM (with $p=0$ on the free-surface) and including the proposed contour matrix, FSMI.

upper part with a density of $2600 \mathrm{~kg} / \mathrm{m}^{3}$. The upper surface of the recipient is open and therefore the upper fluid has a free-surface. The viscosity of both fluids was fixed to 1.00 Pas. Owing to the difference in density instability occurs and the lighter fluid moves towards the free-surface reaching it after $3.8 \mathrm{~s}$. Figure 11 shows the initial position of both fluids and the position after $3.8,10$ and $15 \mathrm{~s}$.

The example was tested using the standard FSM and then with the new FSMI. Figure 12 shows the total volume with both formulations at different time steps. During the first $3.8 \mathrm{~s}$ both fluids move and mix but the free-surface remains nearly stable. This is the reason why during the first $3.8 \mathrm{~s}$ there is not any appreciable volume variation for none of both methods. Once the lighter fluid reaches the free-surface, a wave is produced and the FSM causes a significant mass loss. The FSMI leads to a nearly zero volume variation.

\section{CONCLUSIONS}

The fractional-step method (FSM) is a very efficient and accurate method for solving the incompressible Navier-Stokes equations via a decoupled equal-order finite element scheme solving iteratively but separately the nodal velocities and the pressure variables. The most efficient scheme is the standard fractional method in which the pressure unknown is solved via a Laplace equation. Unfortunately, for free-surface flows this algorithm loses mass near the free-surface and, therefore, 
the algorithm is useless. In this paper we have shown that adding a simple mass-type boundary matrix around the free-surface makes the loss of mass negligible, while the algorithm keeps all the advantages of the standard FSM.

\section{ACKNOWLEDGEMENTS}

The authors thank Monica De Mier for performing some of the numerical tests. This work was also supported by the SEDUREC project of the Consolider Injenio 2010 programme of the Minister of Science and Education of Spain.

\section{REFERENCES}

1. Chorin AJ. The numerical solution of the Navier-Stokes equations for an incompressible fluid. AEC Research and Development Report, NYO-1480-82, New York University, New York, 1967.

2. Chorin AJ. Numerical solution of the Navier-Stokes equations. Mathematics of Computation 1968; 22:745-762.

3. Codina R, Blasco J. A finite element formulation for the Stokes problem allowing equal velocity-pressure interpolation. Computer Methods in Applied Mechanics and Engineering 1997; 143:373-391.

4. Codina R, Blasco J. Analysis of a pressure stabilized finite element approximation of the stationary Navier-Stokes equations. Numerische Mathematik 2000; 87:59-81.

5. Codina R, Vazquez M, Zienkiewicz OC. A general algorithm for compressible and incompressible flow-Part III. The semi implicit form. International Journal for Numerical Methods in Fluids 1998; 27:13-32.

6. Limache A, Idelsohn SR, Rossi R, Oñate E. The violation of objectivity in Laplace formulations of the Navier-Stokes equations. International Journal for Numerical Methods in Fluids 2007; 54(6-8):639-664.

7. Idelsohn SR, Oñate E, Del Pin F. The particle finite element method: a powerful tool to solve incompressible flows with free-surfaces and breaking waves. International Journal for Numerical Methods in Engineering 2004; 61:964-989.

8. Idelsohn SR, Oñate E, Del Pin F. A lagrangian meshless finite element method applied to fluid-structure interaction problems. Computers and Structures 2003; 81:655-671.

9. Hughes TJR, Franca LP, Balestra M. A new finite element formulation for computational fluid dynamics: V. Circumventing the Babuska-Brezzi condition: a stable Petrov-Galerkin formulation for the Stokes problem accommodating equal order interpolations. Computer Methods in Applied Mechanics and Engineering 1986; 59:85-99.

10. Oñate E. Possibilities of finite calculus in computational mechanics. International Journal for Numerical Methods in Engineering 2004; 60(1):255-281.

11. Aubry R, Idelsohn SR, Oñate E. Fractional step like schemes for free surface problems with thermal coupling using the Lagrangian PFEM. Computational Mechanics 2006; 38(4-5):294-309.

12. Gresho PM, Sani RL. Incompressible Flow and the Finite Element Method. 2 Volume Set. Wiley: West Sussex, U.K., 2000. 